\title{
Autoantigens are translocated into small apoptotic bodies during early stages of apoptosis
}

\author{
M Schiller ${ }^{*, 1}$, I Bekeredjian-Ding ${ }^{2}$, P Heyder ${ }^{1}, \mathrm{~N} \mathrm{Blank}^{1}, \mathrm{AD} \mathrm{Ho}^{1}$ and H-M Lorenz ${ }^{1}$
}

A dysregulation of apoptosis or an ineffective clearance of apoptotic material is suspected to be involved in the pathogenesis of systemic lupus erythematodes. Subcellular fragments such as apoptotic bodies (ABs) have been recognized as modulators of intercellular communication and immune function. In this context, we have been interested whether nuclear and cytoplasmic antigens are relocated into $A B s$. In the present study, we characterized $A B s$ isolated from apoptozing lymphoblasts. We found an accumulation of the linker-histone (histone 1) as well as the core-histones (histone 2A, histone 2B, histone 3, histone 4) in ABs. Further, they contained DNA, RNA and the ribonuclear protein La/SSB. Proteins such as cytochrome $c$, HSP 70, prohibitin, p53, nuclear matrix antigen or lamin B were excluded from ABs. The content of ABs differed from that observed in membrane microparticles isolated from viable cells. Formation of ABs occurred early during apoptosis. It was observed before DNAdegradation or phosphatidylserine exposure was detected. ABs were engulfed by monocyte-derived phagocytes. These findings suggest that immunogenic molecules are actively translocated into ABs followed by a rapid engulfment of the latter by environmental phagocytes. In autoimmune diseases, a defect in the clearance of $A B s$ or $A B$ formation may contribute to the development of autoimmunity.

Cell Death and Differentiation (2008) 15, 183-191; doi:10.1038/sj.cdd.4402239; published online 12 October 2007

Autoimmune diseases such as systemic lupus erythematodes (SLE) are characterized by the occurrence of autoantibodies against various, mostly nuclear antigens. To date, the mechanisms leading to this autoantibody formation are not completely understood. Nevertheless, several publications released during the previous years point out that a dysregulation of apoptosis or an ineffective clearance of apoptotic cells contributes to the development of autoimmunity. ${ }^{1-4}$ Our group and others have shown an increased rate of apoptosis in patients suffering from SLE. Further, we previously demonstrated that cells obtained from SLE patients show a hyporesponsiveness to survival factors and are therefore more susceptible to apoptosis than cells of healthy individuals. ${ }^{3,5,6}$ Furthermore, an impaired engulfment of apoptotic material by macrophages obtained from SLE patients has been described..$^{7,8}$ On the background of these data, it seems likely that an increased rate of apoptosis and an ineffective clearance of apoptotic material could result in a breakdown of self-tolerance and an autoimmune response against autoantigens contained in apoptotic particles. Accumulation of apoptotic material may cause a continuous stimulation of potential autoreactive cells leading to chronic autoimmunity and the development of chronic inflammation in SLE patients.

Nuclear antigens seem to play a central role in the pathogenesis of SLE (e.g. antibodies against histones or DNA). Thus, knowledge about the occurrence and localization of these antigens in apoptotic cells is essential for our understanding of how dysregulation of apoptosis or clearance can contribute to the development of autoimmunity. In viable cells, nuclear antigens cannot easily be accessed as the nucleus is well covered by the outer cell membrane, the cytoplasm and the nuclear envelope. Previously, we have already shown an accumulation of nuclear antigens in the cytoplasm of apoptotic lymphocytes. ${ }^{9}$ It has been described that nucleosomes are exposed on the cellular surface during apoptosis. ${ }^{10}$ This autoantigen translocation can enhance phagocytosis of apoptotic material and presentation of autoantigens. ${ }^{11}$ Thus, antigens leave the nucleus during apoptosis and might get accessible for immune cells.

Subcellular fragments have been recognized as mediators of intercellular communication and modulators of immune function. Membrane microparticles (MMP) are coated vesicles, which are released from a variety of cells. This shedding is mainly observed during cellular activation or apoptosis. ${ }^{12-14}$ These subcellular fragments have previously been shown to indirectly activate CD4 + lymphocytes. ${ }^{15,16}$ Further, apoptotic bodies (ABs) have been identified as B-cell autoantigens. ${ }^{17}$ The process of $A B$ formation has been investigated by several groups during the previous years and surface blebs of various size and content have been described. ${ }^{18-21}$ Surface blebs separate from the cellular remnant and convert into smaller ABs. Throughout this paper, subcellular particles that have

\footnotetext{
${ }^{1}$ Department of Medicine V, University of Heidelberg, Im Neuenheimer Feld 410, Heidelberg, Germany and ${ }^{2}$ Department of Medical Microbiology and Hygiene, University of Heidelberg, Im Neuenheimer Feld 324, Heidelberg, Germany

${ }^{*}$ Corresponding author: M Schiller, Department of Medicine V, University of Heidelberg, Im Neuenheimer Feld 410, 69120 Heidelberg, Germany.

Tel: + 49622156 38841; Fax: + 49622156 5772; E-mail: Martin.Schiller@med.uni-heidelberg.de

Keywords: apoptosis; apoptotic bodies; autoantigens; histones

Abbreviations: ABs, apoptotic bodies; CFSE, carboxyfluorescein succinimidyl ester; DiOC6, 3,3-dihexyloxacarbocyaniniodode; FSC/SSC, forward scatter/side scatter; $\mathrm{HSP}$, heat shock protein; $\mathrm{H} 1$, histone 1 ; $\mathrm{H} 2 \mathrm{~A}$, histone $2 \mathrm{~A}$; $\mathrm{H} 2 \mathrm{~B}$, histone $2 \mathrm{~B}$; $\mathrm{H} 3$, histone 3; $\mathrm{H} 4$, histone 4; $\mathrm{MetOH}$, methanol; ML-9, myosin light chain kinase inhibitor; MMP, membrane microparticles; NUMA, nuclear matrix antigen; PBMC, peripheral blood mononuclear cells; PI, propidium iodide; PS, phosphatidylserine; SLE, systemic lupus erythematodes; STS, staurosporine; Y-27632, rho-kinase inhibitor; zVAD-FMK, pan-caspase inhibitor

Received 29.5.07; revised 20.8.07; accepted 06.9.07; Edited by B Osborne; published online 12.10.07
} 
separated from apoptozing cells are referred to as ABs. Particles separated from activated or living cells are called MMP. To strictly avoid contamination of isolated microparticles and ABs by apoptotic cells and cellular remnants, we focussed within this study on $A B s$ of a size smaller than $1.2 \mu \mathrm{m}$.

$A B$ formation and a rapid engulfment of $A B$ s by environmental phagocytes ensure an anti-inflammatory clearance of apoptotic debris under physiological conditions. ${ }^{22-24}$ The aim of the present study was to gain insight into the physiology of $A B$ formation and into molecular composition of ABs. Further, we have been interested whether $A B$ s are effectively engulfed by phagocytes.

\section{Results}

Apoptotic cell blebbing occurs time and dose dependently after apoptosis induction and is inhibited by inhibitors of rho-kinase, myosin light chain kinase and caspases. To analyze $A B$ formation, we induced apoptotic cell death in human lymphoblasts by UV-B irradiation. After induction of apoptosis, cells and ABs were harvested as described and analyzed by flow cytometry (for details see Material and methods). We could detect an increase of subcellular fragments already at $2 \mathrm{~h}$ after induction of apoptosis (Figure 1). The population of subcellular fragments was clearly separated from the cellular population. As shown in Figure 2a, we detected an even more pronounced increase of subcellular fragments after staurosporine (STS) treatment, compared to UV-Birradiated cells. The increase of subcellular fragments after apoptosis induction turned out to be time dependent (Figure 2a). We further observed a dose-dependent increase in $A B$ formation after application of varying doses of UV-B (not shown). Induction of necrosis by treatment with hydrogen peroxide $\left(\mathrm{H}_{2} \mathrm{O}_{2}\right)$, methanol (MetOH) or by heating the cells at $56^{\circ} \mathrm{C}$ for $30 \mathrm{~min}\left(56^{\circ} \mathrm{C} 30 \mathrm{~min}\right)$ did not lead to an increased formation of subcellular fragments (Figure 2a). A

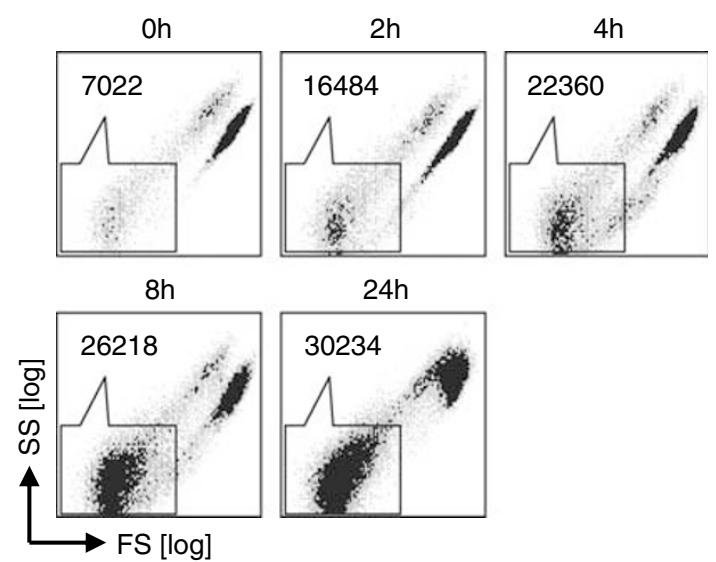

Figure 1 Subcellular fragments containing ABs and MMP can be separated from apoptotic and viable cells by flow cytometry. Dot plots show FSC/SSCproperties of apoptotic cells and subcellular fragments (circled population) after induction of apoptosis by UV-B irradiation $(90 \mathrm{~mJ} / \mathrm{cm})$. Subcellular fragments were quantified after the indicated incubation periods background level of subcellular fragments (MMP) was detected in samples. In contrast to apoptozing cells, we did not observe a time-dependent increase of subcellular fragments in viable or necrotic cells (Figure 2a).

Light microscopic analyses of viable, apoptotic and necrotic cells were performed to confirm flow cytometric data. Consistently, we observed an increased membrane blebbing after apoptosis induction (UV-B, STS), whereas viable or necrotic cells (w/o, $\mathrm{H}_{2} \mathrm{O}_{2}, \mathrm{MetOH}, 56^{\circ} \mathrm{C} 30 \mathrm{~min}$ ) showed no increased membrane blebbing. Figure $2 \mathrm{~b}$ shows microscopic pictures of viable, apoptotic and necrotic cells after 4 and $8 \mathrm{~h}$ of incubation.

Comparing ABs with standard sized micro beads, we could define the average size of these isolated microparticles to about $500 \mathrm{~nm}$ (Figure 3a). Finally, isolated ABs were characterized by transmission electron microscopy. Electron microscopy confirmed that isolated ABs are membranecoated vesicles of an average size of about $500 \mathrm{~nm}$ (Figure 3b).

Shedding of $A B s$ from dying cells might require reorganization of the cytoskeleton. Thus, we have been interested, whether $A B$ formation can be influenced by inhibitors of cytoskeletal activation or by inhibition of caspases. We used inhibitors of rho-kinase (Y-27632), myosin light chain kinase (ML-9) or the pan-caspase inhibitor (zVAD-FMK). ${ }^{18-21}$ The use of these inhibitors revealed that formation of $A B s$ depends on the activation of the apoptotic signaling cascade (caspase activation) and the consecutive cytoskeletal activation. Formation of $A B s$ was significantly inhibited by $\mathrm{Y}-27632$ $(27.7 \pm 1.8 \%)$, ML-9 $(59.0 \pm 10.1 \%)$ and most efficiently by zVAD-FMK (99.0 $\pm 6.7 \%)$ (Figure 4).

Taken together, we observed an increased formation of subcellular fragments referred to as $A B s$ after induction of apoptosis. $A B$ formation occurred time and dose dependently and it was inhibited by blocking both caspase activation and activation of cytoskeletal molecules, respectively.

Autoantigens are translocated into ABs. Next, we investigated whether nuclear antigens can be detected in isolated $A B s$ and whether we can detect any molecular differences between $\mathrm{ABs}$ and MMP released form activated non-apoptotic lymphoblasts. ABs were isolated from the supernatant of UV-B-irradiated or STS-treated cells and MMP were obtained from the supernatant of viable (untreated) cells. Antigens were detected by Western blot analysis. We observed an increased content of the histones, histone $1(\mathrm{H} 1)$, histone $2 \mathrm{~A}(\mathrm{H} 2 \mathrm{~A})$, histone $2 \mathrm{~B}(\mathrm{H} 2 \mathrm{~B})$, histone 3 $(\mathrm{H} 3)$ and histone $4(\mathrm{H} 4)$, in lysates of $A B s$ compared to lysates of MMP isolated from viable cells (Figure 5 ). The detection of a doublet in H2B blots is probably caused by post-translational modifications of this protein as histones are extensively modified during apoptosis. ${ }^{25,26}$ The ribonuclear protein La/SSB, another well-known autoantigen, was also translocated into ABs (Figure 5). Beside these proteins, DNA and RNA are common autoantigens in autoimmune disease. Both were present in $A B s$ in a ratio of 10:1 (DNA : RNA), and a two- to three-fold higher concentration of DNA was detected in ABs compared to MMP. By gel electrophoresis, we observed that DNA in ABs was degraded to fragments of about 500-1000 bp (not shown). 
a

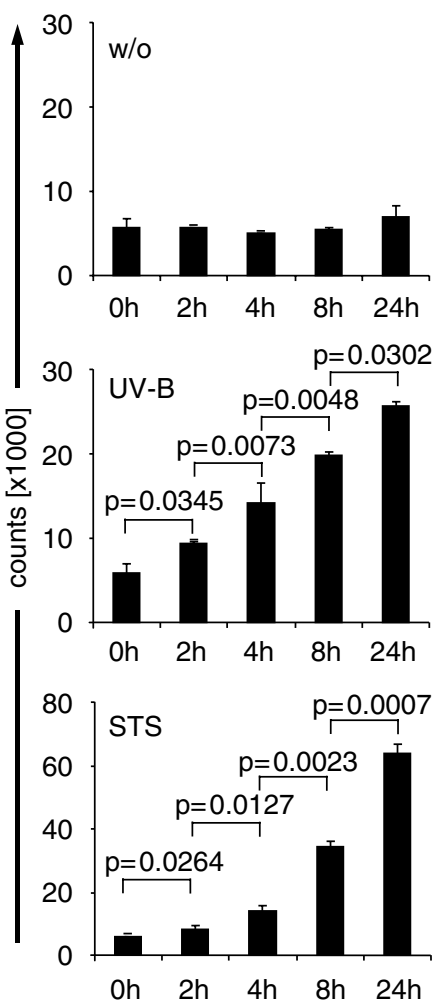

30

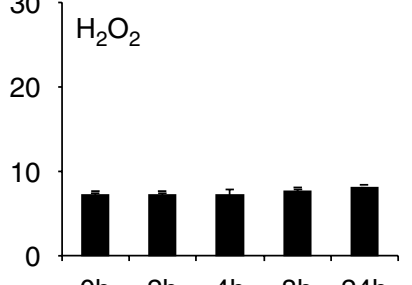

Oh $2 \mathrm{~h} \quad 4 \mathrm{~h} \quad 8 \mathrm{~h} \quad 24 \mathrm{~h}$
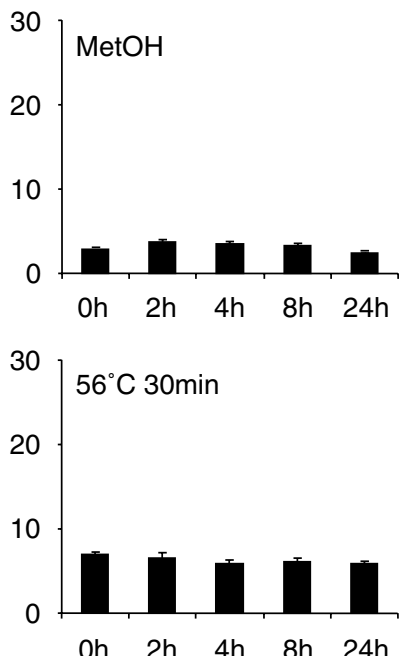

b
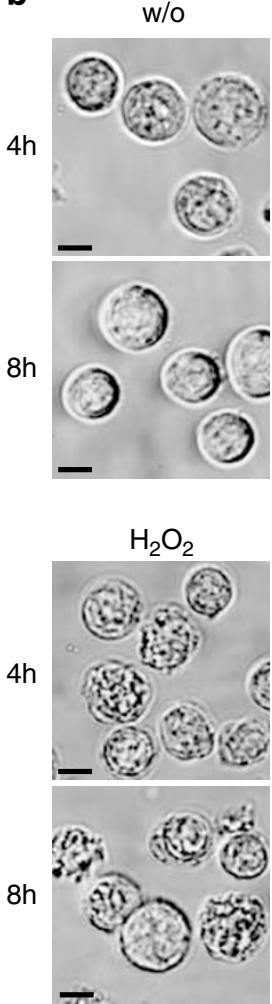

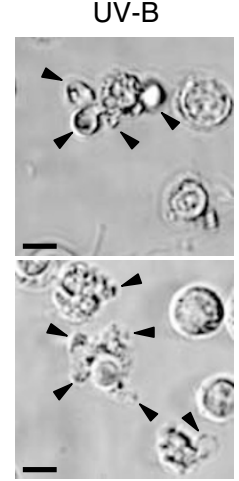

STS
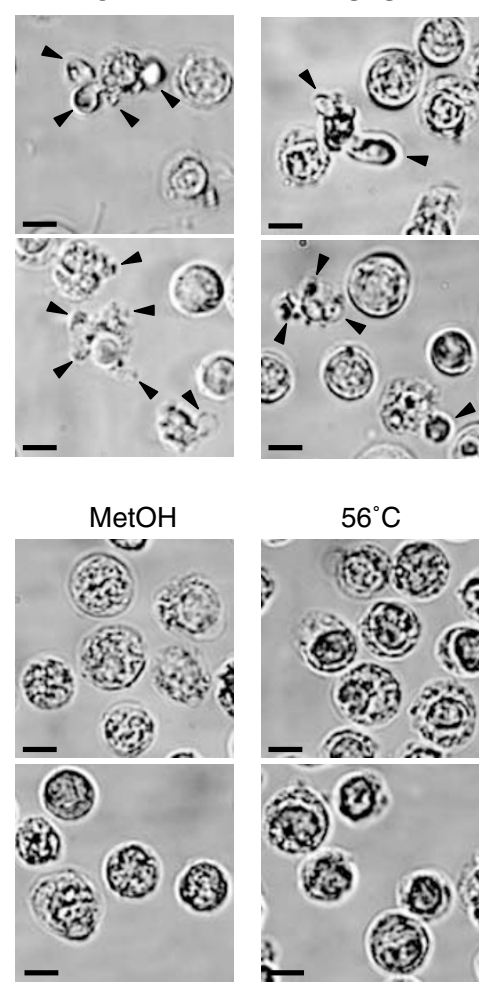

Figure 2 (a) Formation of ABs occurs time dependently after apoptosis induction. Activated human lymphoblasts were induced to undergo apoptosis by UV-B irradiation $(90 \mathrm{~mJ} / \mathrm{cm})(\mathrm{UV}-\mathrm{B})$ or STS treatment $(1 \mu \mathrm{M})$. Necrosis was induced in control samples by heating cells at $56^{\circ} \mathrm{C}$ for $30 \mathrm{~min}\left(56^{\circ} \mathrm{C} 30 \mathrm{~min}\right)$, by treatment with MetOH $(50 \%$ for 15 min on ice), or by treatment with $\mathrm{H}_{2} \mathrm{O}_{2}(100 \mu \mathrm{M})\left(\mathrm{H}_{2} \mathrm{O}_{2}\right)$. After the indicated incubation periods (x-axis), the amount of ABs was quantified by flow cytometry. On the $y$ axis, the amount of subcellular fragments is shown (counts). Untreated cells were analyzed as control (w/o). Standard deviations are indicated in the figure and statistical significance ( $P$-value) was calculated by Student's t-test. Data were obtained from three independent experiments. (b) Cells were induced to undergo apoptosis or necrosis as described above. Then analysis by light microscopy was performed (4 and $8 \mathrm{~h}$ after treatment). Membrane blebbing was to be observed in UV-B- and STS-treated cells (indicated by black arrow tips). In contrast, untreated or necrotic cells show a round morphology and no shedding of membrane vesicles on their surface. Black bars indicate $5 \mu \mathrm{m}$

Taken together, autoantigens are concentrated in ABs after induction of cell death by UV-B irradiation or by STS treatment, whereas in MMP released from activated cells, only a low amount of autoantigens (or no antigen at all) was detected. It is important to note that we used the same amount of protein analyzing samples obtained from $A B s$ and samples obtained from MMP. Thus, the increase of antigens within ABs is not due to a more intense cell blebbing, but is rather caused by an active translocation of these antigens into $A B s$ and an altered molecular composition of the latter.

Distribution of antigens between cytoplasm and ABs during programmed cell death. To characterize the distribution of further structural and cytoplasmic molecules during apoptosis, we analyzed cytoplasmic lysates of viable and apoptotic cells, ABs and MMP. Cytochrome $c$, a mitochondrial molecule, which is released from mitochondrial intermembrane space during apoptosis, was not translocated into ABs. Similar results were obtained for heat shock protein (HSP) 70 and prohibitin. p53 content markedly increased in cytoplasmic preparations after apoptosis induction, whereas in $\mathrm{ABs}$, no signal was detected at the molecular weight of $53 \mathrm{kDa}$. The nuclear proteins nuclear matrix antigen (NUMA) and Lamin B were not translocated into $A B s$ and we observed a decreased cytoplasmic protein content of Lamin B, most likely caused by a degradation of the protein during apoptosis (Figure 6).

Time kinetic of apoptotic membrane changes, DNA degradation and translocation of proteins into apoptotic blebs. We performed flow cytometry after varying incubation periods $(0,2,4,8$ and $24 \mathrm{~h})$ to evaluate a time correlation between well-known hallmarks of apoptosis and formation of ABs. Phosphatidylserine (PS) exposure on the outer membrane leaflet and the percentage of cells showing hypodiploid DNA content (Sub G1 peak) were analyzed by Annexin $\mathrm{V}$ staining and propidium iodide (PI) staining in the presence of detergent. Analysis of mitochondrial membrane potential was carried out by staining the cells with the fluorochrome 3,3-dihexyloxacarbocyaniniodode (DiOC6). In parallel, we performed Western blot analysis to detect the histone H2B. We observed a significant increase of cells that stained positive for Annexin V or were characterized by hypodiploid 

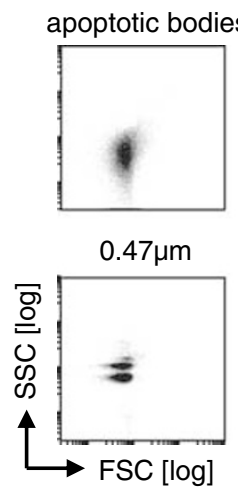

b

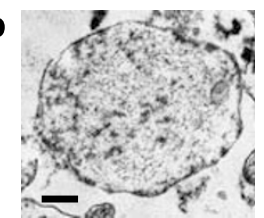

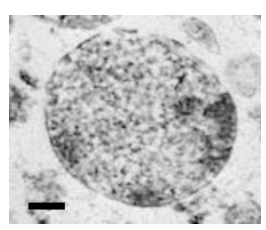

PBS

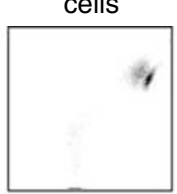

$1 \mu \mathrm{m}$
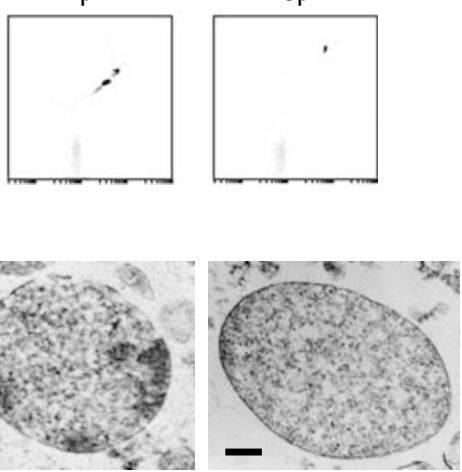

Figure 3 (a) ABs and MMP are isolated by filtration and ultracentrifugation and their size can by determined to about $500 \mathrm{~nm}$. After induction of apoptosis, subcellular fragments were isolated by filtration and ultracentrifugation. Thereafter, isolated cells and subcellular fragments were analyzed by flow cytometry. In the upper dot plots, FSC/SSC properties of ABs (left) and cells (middle) are shown. The right dot plot shows a control (PBS) to exclude any contamination during cytometry. The lower dot plots show standard-sized micro beads $(0.47,1.0,2.8 \mu \mathrm{m})$, which were analyzed simultaneously. (b) Transmission electron microscopy of isolated $A B s$ reveals that isolated subcellular fragments are membrane-coated vesicles. Consistently with flow cytometric analysis, their size was determined to about $500 \mathrm{~nm}$ (Black bar: $100 \mathrm{~nm}$ ). Three representative sections are shown

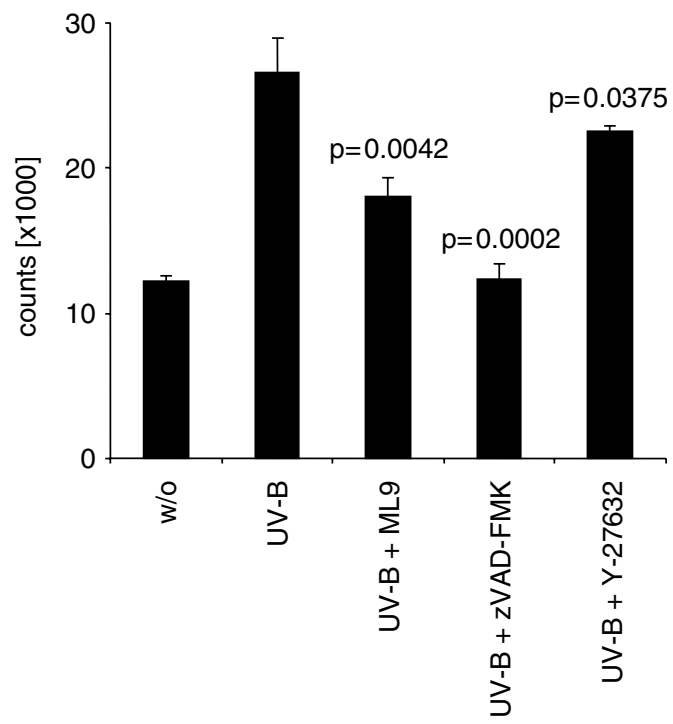

Figure $4 A B$ formation is inhibited by ML-9, zVAD-FMK and Y-27632. Apoptosis was induced in activated lymphoblasts by UV-B irradiation $\left(90 \mathrm{~mJ} / \mathrm{cm}^{2}\right)$ in presence of ML-9, ZVAD-FMK and Y-27632. After incubation for $6 \mathrm{~h}$, the amount of $A B s$ was quantified by flow cytometry. Counts of subcellular fragments are shown ( $P$-values to UV-B alone as indicated). ML-9, ZVAD-FMK and Y-27632 significantly inhibited $A B$ formation. Data were obtained from four independent experiments and statistical significance $(P$-value) was calculated by Student's $t$-test. Standard deviations are indicated

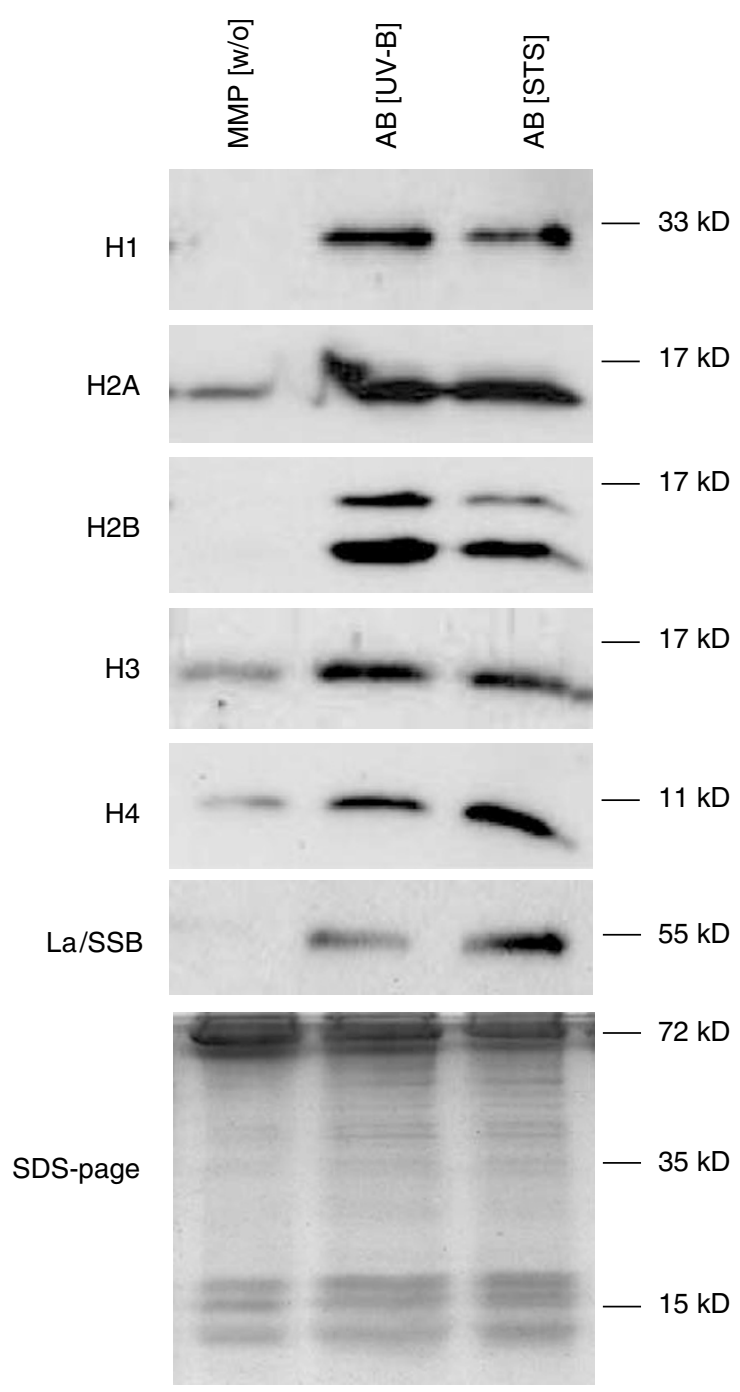

Figure 5 Autoantigens are translocated into $A B s$. MMP isolated from viable cells (MMP (w/o)) and ABs isolated from UV-B-irradiated $\left(90 \mathrm{~mJ} / \mathrm{cm}^{2}\right.$ ) (ABs (UV-B)) or STS-treated cells $(1 \mu \mathrm{M})$ (ABs (STS)) were harvested and resuspended in lysis buffer. Then, protein electrophoresis and Western blot analysis were performed as described in Material and Methods. The detected proteins are indicated in the figure. The lowest panel shows a total protein SDS-PAGE analysis of lysates obtained from MMP and $A B s$

DNA content after $8 \mathrm{~h}$ of incubation. In contrast to this, a significant increase of $A B$ formation and a marked increase of $\mathrm{H} 2 \mathrm{~B}$ protein content within $\mathrm{ABs}$ were detected already after $4 \mathrm{~h}$.

Very early during apoptotic cell death, a breakdown of the inner mitochondrial transmembrane potential is to be observed. ${ }^{27,28}$ Analyzing mitochondrial membrane potential, we noticed a significant decrease of the mitochondrial membrane potential at $4 \mathrm{~h}$ after UV-B irradiation. After $24 \mathrm{~h}$, nearly all cells showed a collapsed mitochondrial membrane potential. Thus, formation of $\mathrm{ABs}$ and translocation of autoantigens into the latter were observed in parallel with a breakdown of the mitochondrial transmembrane potential and preceded other classical hallmarks of apoptosis such as PS exposure or DNA degradation (Figure 7). 


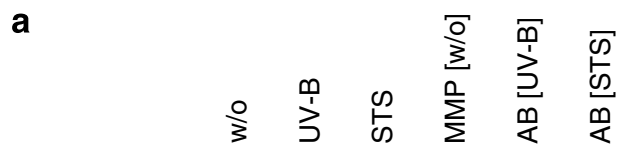

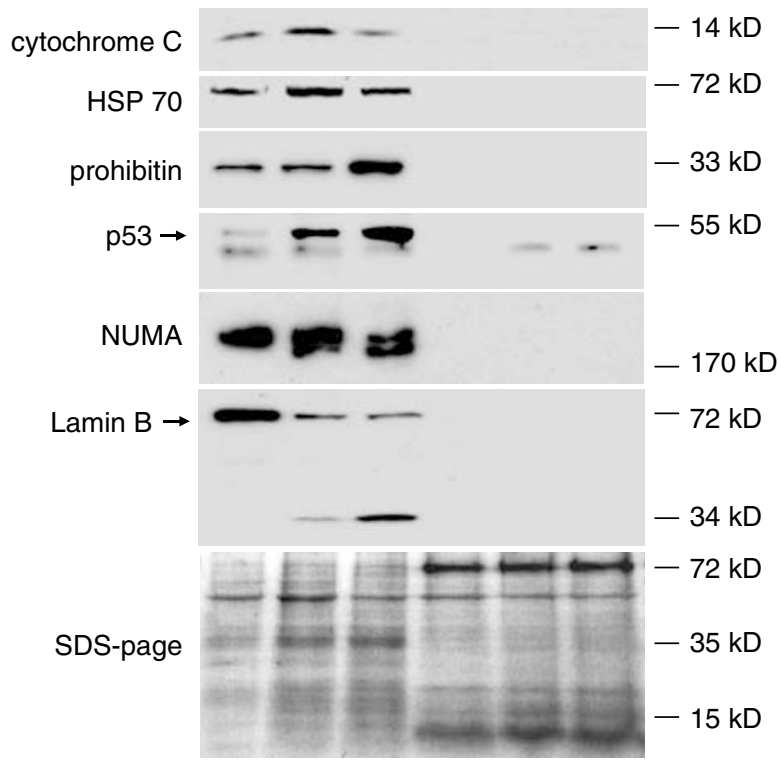

b

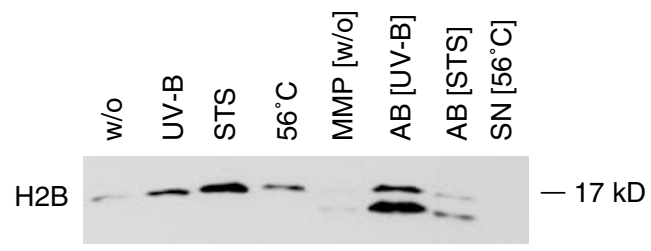

Figure 6 Distribution of proteins between cytoplasm, MMP and ABs. (a) Proteins were detected in cytoplasmic lysates obtained from viable (w/o) and apoptotic cells (UV-B $90 \mathrm{~mJ} / \mathrm{cm}^{2}$, STS $1 \mu \mathrm{M}$ ) by Western blot analysis. In parallel, lysates of isolated MMP(w/0) and $A B s$ (ABs (UV-B), ABs (STS)) were analyzed. Cytochrome c, HSP 70, prohibitin, p53, lamin B and NUMA were detected. The lowest panel shows a total protein SDS-PAGE analysis of the prepared lysates. (b) $\mathrm{H} 2 \mathrm{~B}$ was detected in viable cells, apoptotic cells, MMP and ABs. As control necrotic cells $\left(56^{\circ} \mathrm{C}\right)$ and supernatants of necrotic cells $\left(\mathrm{SN}\left(56^{\circ} \mathrm{C}\right)\right)$ were analyzed

ABs are engulfed by monocyte-derived phagocytes. ABs labeled by carboxyfluorescein succinimidyl ester (CFSE) were co-incubated with monocyte-derived phagocytes to analyze the engulfment of $A B s$ by the latter. Gating on monocyte-derived phagocytes, we analyzed mean fluorescence intensity (MFI) after the indicated incubation periods. The amount of ABs was quantified by forward scatter/side scatter properties (FSC/SSC) analysis. We observed an increasing fluorescence (gating on phagocytes) indicating an engulfment of CFSE-labeled ABs. Simultaneously, a decreased amount of $A B s$ was detected (Figure 8a). To discriminate between mere binding of $A B s$ to phagocytes and actual engulfment of $A B s$ by the latter, confocal microscopy was performed. Analyzing the co-cultures of phagocytes and $A B s$ at $0 \mathrm{~h}$ of incubation, $A B s$ were found outside the phagocytes. With prolonged incubation times, we observed a marked increase of $A B s$ that were localized inside the cells. Thus, ABs were in fact engulfed by monocyte-derived phagocytes (Figure 8b).

\section{Discussion}

Apoptotic cell blebbing and formation of ABs are hallmarks of apoptosis. During apoptosis, cellular components are packed within membrane vesicles (apoptotic blebbing) and antigens are relocalized towards the surface of the cell. ${ }^{10,11}$ Thereafter, these membrane vesicles separate from cellular remnant as ABs. Apoptotic blebbing plays a central role for the interaction of a dying cell with the immune system and MMP released from viable cells as well as ABs seem to be mediators of intercellular communication. ${ }^{29}$ During apoptosis, a multiplicity of $A B s$ separates from the dying cell. These $A B s$ then can interact with surrounding cells or are engulfed by phagocytes. Other groups previously reported that an uptake of ABs could lead to an immunogenic presentation of self-antigens in an inflammatory context. Activation of various cell types as a consequence of impaired clearance of apoptotic material cells has been repeatedly described during the previous years ${ }^{11,30}$ and it has been shown that autoantigens such as ribonuclear proteins, histones or DNA are exposed on the surface of apoptozing cells and ABs. ${ }^{10,17,31}$ Further, we have recently observed that $A B$ s stimulate plasmacytoid dendritic cells to produce type-1-interferon. ${ }^{32}$

In the present study, we wanted to characterize the mechanisms leading to $A B$ formation using primary isolated human cells. We analyzed the molecular composition of ABs that already have been separated from the cellular remnant and investigated which antigens are translocated into $A B s$ and whether some antigens cannot be detected within them. As a cellular model, we used activated lymphoblasts, which are highly susceptible to apoptotic cell death. ${ }^{33,34}$

First, we analyzed pathways involved in $A B$ formation. We observed that apoptotic cell blebbing was dependent on the activation of caspases as well as the activation of cytoskeletal molecules. In these experiments, we used inhibitors of Y-27632, ML-9 and a zVAD-FMK. Formation of ABs occurred dose and time dependently after apoptosis induction. However, we did not observe any increase in the formation of subcellular fragments analyzing viable or necrotic cells. Taken together, the observed increase in subcellular fragments (ABs) after induction of apoptosis is clearly caused by apoptotic cell blebbing and the consecutive formation of $A B s$.

Analyzing lysates of isolated ABs, we detected autoantigens such as histones as well as the ribonuclear protein La/SSB. Further, DNA fragments of 500-1000 bp size were observed within ABs. The lysates of MMP (obtained from viable cells) contained only marginal amounts of these antigens. This finding is consistent with observations by Théry et al. $^{35}$ who demonstrated that MMP (exosomes released by dendritic cells) are biochemically and morphologically different from ABs. The molecules found in ABs are well known autoantigens in $\operatorname{SLE}^{36}$ and interestingly, other cytoplasmatic molecules such as cytochrome $c$, prohibitin and HSP 70, lamin B and NUMA were not detected in ABs. These data suggest that antigens are not randomly packed into ABs. Rather, it seems likely that apoptozing cells actively regulate the translocation of molecules into ABs. In a previous study, we have shown a cytoplasmic accumulation of histones during apoptotic cell death, which was caused by a translocation of these molecules and not by a de novo protein synthesis. ${ }^{9}$ 

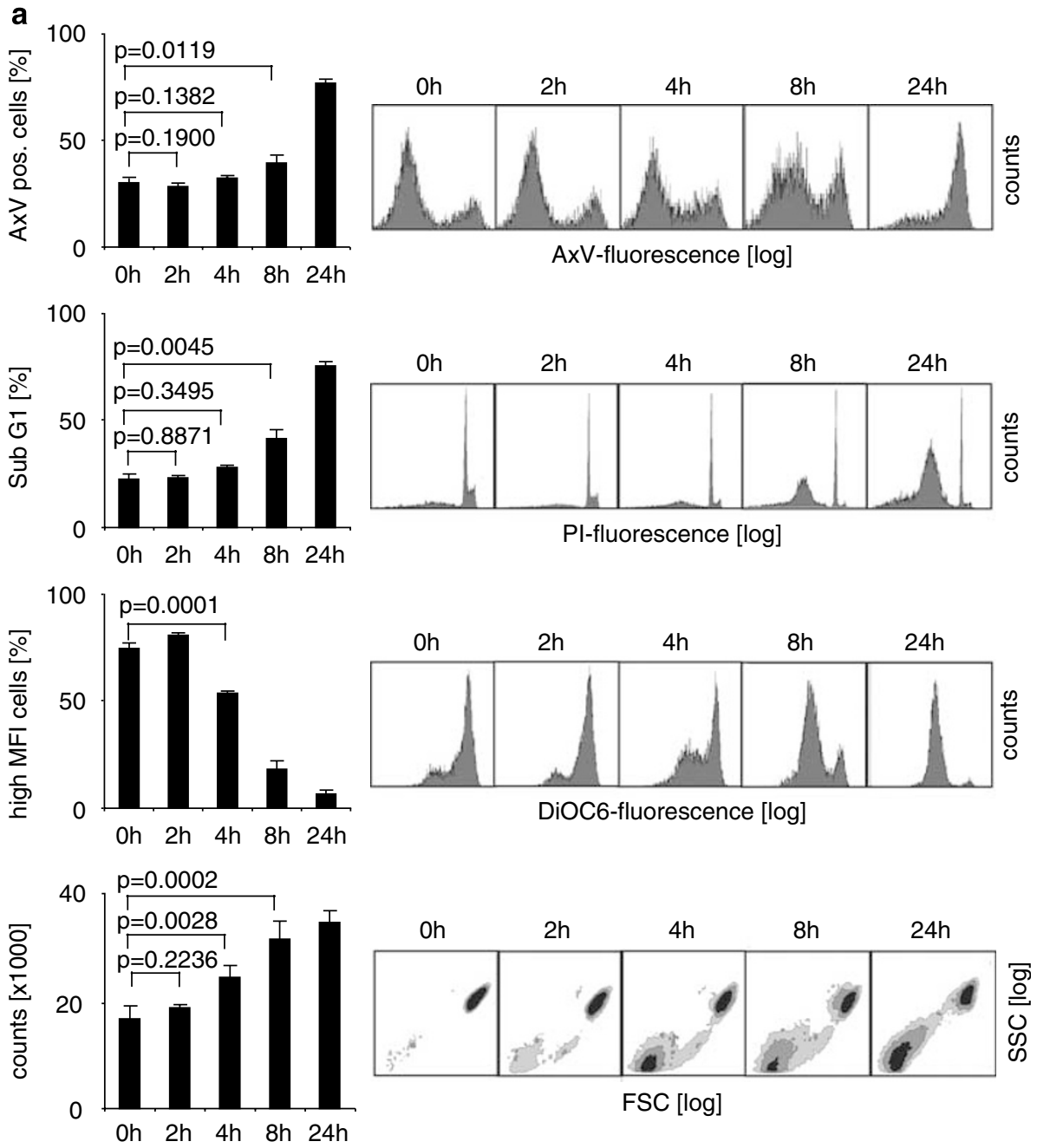

b

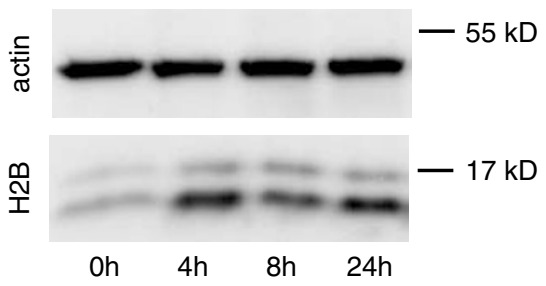

Figure 7 ABs rapidly detach from apoptozing cells and antigens are translocated into ABs early after apoptosis induction. (a) After induction of apoptosis by UV-B irradiation, we quantified the percentage of cells showing a PS exposure (AxV pos. cells) or containing hypodiploid DNA (Sub G1). Further, the percentage of cells with high mitochondrial membrane potential was quantified. These cells show high MFI after DiOC6 staining. In parallel, the amount of ABs was quantified (counts). FACS analysis was performed after the indicated incubation periods. Data shown in the left graphs were obtained from four independent experiments and statistical significance $(P$-value $)$ was calculated by Student's $t$-test. Standard deviations are indicated. The right panel shows primary data of one representative experiment. (b) Lysates of ABs were prepared after the indicated incubation periods and the histone H2B was detected by Western blot analysis. The upper Western blot shows the corresponding actin. One representative experiment is shown

Autoantigens were translocated into $A B s$ early after apoptosis induction and they were found in $A B s$ even before other hallmarks of apoptosis such as DNA degradation or PS exposure were observed. During apoptosis, a multiplicity of $A B s$ is shed from the dying cell. To avoid an inappropriate immune stimulation, $A B s$ have to be cleared effectively by environmental phagocytes. Based on this concept, we analyzed the engulfment of $A B s$ by monocyte-derived phagocytes. In fact, we detected an engulfment of $A B s$ by phagocytes, starting already after $2 \mathrm{~h}$ of co-incubation. Taken together, we herein demonstrate that autoantigens are translocated into small $A B s$ during early stages of apoptosis. These antigens are well known from autoimmune diseases such as SLE. The molecular content of ABs is clearly different from the one observed in MMP isolated from viable cells. ABs rapidly detach from apoptozing cells. They were loaded with 

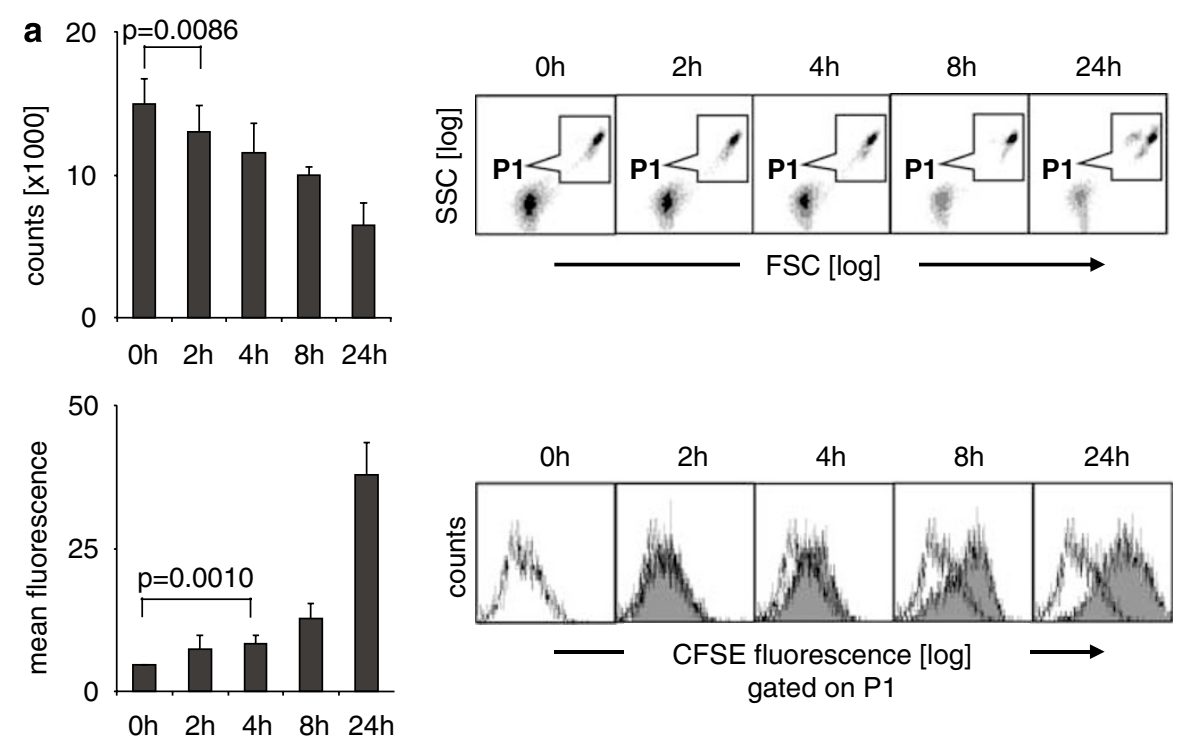

b

Oh

$2 \mathrm{~h}$

$4 \mathrm{~h}$
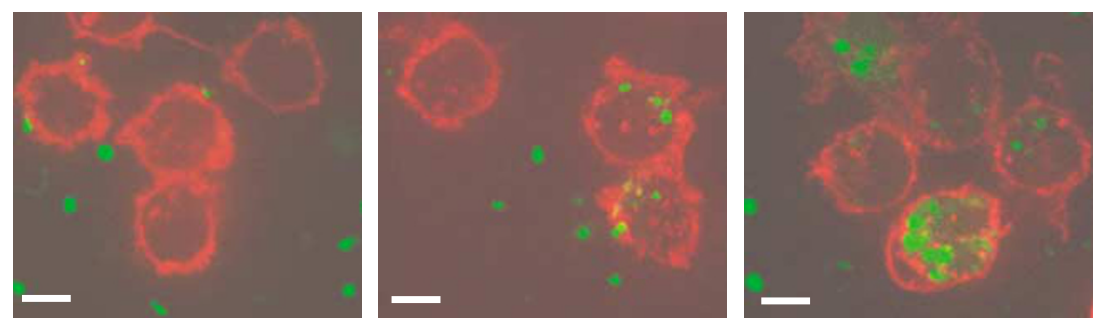

$8 \mathrm{~h}$

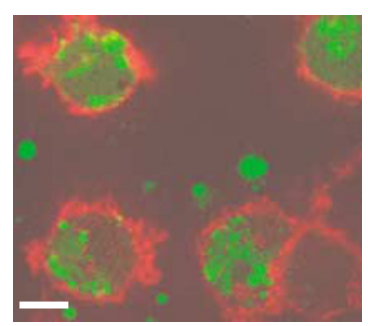

$24 \mathrm{~h}$

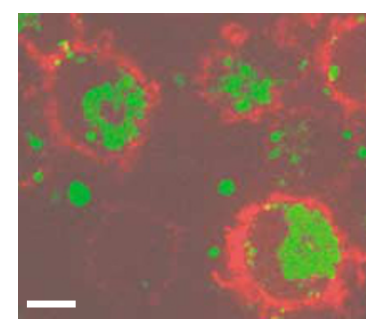

Figure 8 ABs are effectively engulfed by monocyte-derived phagocytes. (a) Monocyte-derived phagocytes (cultured for $24 \mathrm{~h}$ ) were co-incubated with CFSE-labeled ABs. The amount of ABs was quantified after the indicated incubation period by FSC/SSC analysis (upper graph and dot plots). MFI gating on phagocytes (population: P1) is shown in the lower panel. Graphs on the left show data obtained from four independent experiments. Statistical significance ( $P$-value) was calculated by Student's $t$-test. Standard deviations are indicated. The dot plots and histograms show primary data of one representative experiment. (b) Confocal microscopy of anti-CD14-satined phagocytes and CFSE-stained ABs is shown. Phagocytes stained by anti-CD14 antibodies are shown in red. CFSE stained ABs are shown in green. One representative section is shown for each time point. White bars indicate $5 \mu \mathrm{m}$

autoantigens already at $4 \mathrm{~h}$ after apoptosis induction and were effectively engulfed by monocyte-derived phagocytes. An inflammatory response against molecules present in ABs might be initiated, if phagocytosis is impaired or if the phagocytic system is overloaded by apoptotic material. Well-known autoantigens were detected within ABs, whereas other cytoplasmic and structural molecules obviously were excluded. Thus, it seems likely that the formation of $A B s$ and an effective clearance of the latter by phagocytes represent central mechanisms for a silent and non-immunogenic clearance of autoantigens. A disruption of this process would therefore contribute to the initiation of autoimmune diseases.

\section{Materials and Methods}

Cell preparation and culture media. Heparinized venous blood was isolated from normal healthy volunteers after informed consent. Peripheral blood mononuclear cells (PBMC) were separated by density gradient centrifugation (LSM 1077) and washed twice with phosphate-buffered saline (PBS). Cells were cultured in RPMI 1640 supplemented with $4 \mathrm{mM} \mathrm{L-glutamine,} 10 \mathrm{mM} \mathrm{HEPES}$ buffer, $100 \mathrm{U} / \mathrm{ml}$ penicillin, $100 \mu \mathrm{g} / \mathrm{ml}$ streptomycin (all reagents obtained from PAA, Pasching, Germany). Furthermore, RPMI 1640 was supplemented with $10 \%$ (v/v) heatinactivated fetal calf serum (Gibco-BRL, Eggenstein, Germany). Cell viability was assessed by trypan blue exclusion test.

Generation of activated lymphoblasts. To generate lymphoblasts, PBMC were activated with $1 \mu \mathrm{g} / \mathrm{ml}$ phytohemagglutinine (Sigma, Taufkirchen, Germany) and $10 \mathrm{U} / \mathrm{ml} \mathrm{IL-2} \mathrm{(Roche,} \mathrm{Mannheim,} \mathrm{Germany)} \mathrm{for} 5$ days. Thereafter, cells were expanded with $10 \mathrm{U} / \mathrm{ml} \mathrm{IL}-2$ another 2 days. Resulting lymphoblasts were 
extensively washed and cultured as indicated. These cells are quite susceptible to apoptosis-inducing stimuli and reflect activated cells as they can be found in patients with chronic inflammatory diseases.

Induction of cell death and detection of apoptosis. Apoptosis was induced by UV-B irradiation or by treatment with STS (Alexis, Grünberg, Germany). For induction of necrosis, cells were heated at $56^{\circ} \mathrm{C}$ for $30 \mathrm{~min}$, treated by $\mathrm{H}_{2} \mathrm{O}_{2}$ $(100 \mu \mathrm{M})$ or $\mathrm{MetOH}(50 \%)$ for $15 \mathrm{~min}$ on ice. $\mathrm{H}_{2} \mathrm{O}_{2}$ and $\mathrm{MetOH}$ were obtained from Sigma (Taufkirchen, Germany). To quantify the rate of apoptosis, cells were analyzed by flow cytometry. To determine nuclear granularity and hypochromicity, DNA staining with PI (Sigma, Taufkirchen, Germany) was performed in the presence of detergent. ${ }^{37}$ In short, $4 \times 10^{5}$ cells were stained with $50 \mu \mathrm{g} / \mathrm{ml} \mathrm{PI}$ in hypotonic fluorochrome solution containing $0.1 \%$ (w/v) sodium citrate and $0.1 \%(\mathrm{v} / \mathrm{v})$ Triton X 100 (Sigma). DNA degradation leading to a sub-G1 peak in PI staining is a hallmark of apoptosis. The percentage of cells showing a sub-G1 peak was determined by flow cytometry.

For detection of PS exposure on the cell surface staining with FITC-conjugated Annexin V (Böhringer, Mannheim, Germany) in combination with PI (AxV/PI) was performed..$^{38}$ The cells were stained for $30 \mathrm{~min}$ at $4^{\circ} \mathrm{C}$ with $200 \mathrm{ng}$ of AxV-FITC and $500 \mathrm{ng} \mathrm{PI}$ in $500 \mu \mathrm{l}$ Ringer's solution. The samples were immediately analyzed by flow cytometry.

Furthermore, cells undergoing apoptosis show morphological changes, which can be detected by flow cytometric analysis of the FSC/SSC. To analyze mitochondrial membrane, potential cells were labeled with the fluorochrome DiOC6 (Sigma) and incubated for $20 \mathrm{~min}$ at $20^{\circ} \mathrm{C}$ in the dark. After incubation, cells were immediately analyzed by flow cytometry.

Flow cytometric analysis. Flow cytometric analyses were performed using an EPICS XL ${ }^{\mathrm{TM}}$ flow cytometer (Coulter, Hialeah, FI, USA), counting at least 20000 events.

Isolation of ABs and MMP. After induction of apoptosis, the cell culture supernatant was collected. Two additional centrifugation steps $(500 \times g, 5 \mathrm{~min})$ were performed in order to remove remaining cells. The supernatant was then passed through a $1.2 \mu \mathrm{M}$ non-pyrogenic, hydrophilic syringe filter. After centrifugation at $100000 \mathrm{~g}$ for $30 \mathrm{~min}$, the pellet containing ABs or MMP was harvested and used for further experiments.

Isolation and detection of RNA, DNA within cells and ABs. To analyze DNA content ABs were harvested and DNA was isolated using Trizol ${ }^{\mathbb{R}}$ Reagent (Invitrogen, Karlsruhe, Germany) following the manufacturer's protocol. DNA was then either quantified by photometry or subjected to a standard agarose gel. RNA was isolated using RNeasy ${ }^{\mathbb{R}}$ Kit (Quiagen, Hilden, Germany) following the manufacturer's instructions. RNA was quantified by photometry.

Immunoblot analysis. Cells and $\mathrm{ABs}$ were harvested and resuspended in lysis buffer (RIPA, ccpro, Neustadt, Germany) containing a protease inhibitor cocktail (complete Mini, Boehringer, Mannheim Germany). Lysis was performed for $30 \mathrm{~min}$ on ice. After centrifugation at $16000 \mathrm{~g}$, the supernatant was carefully removed and transferred into a new microfuge tube. Protein content of the samples was estimated by BCA assay (Perbio, Bonn, Germany). Each sample $(20 \mu \mathrm{g})$ was diluted in loading buffer and subjected to a standard SDS-PAGE. After transfer to PVDF membranes, proteins were detected using the following antibodies:

Anti-histone H1 (FL-219), anti-histone H3 (FL-136), anti-histone H4 (F-9), antiLa/SSB (N-20), anti-HSP 90 and anti-HSP 70 (all Santa Cruz, Heidelberg, Germany); anti-histone H2A (rabbit antiserum), anti-histone H2B (rabbit polyclonal IgG) (both Upstate/Biomol, Hamburg, Germany); anti-lamin B, anti-NUMA (204-41) (both Calbiochem, Darmstadt, Germany); anti-p53 (Chemicon, Hofheim, Germany); anti-actin (Sigma, Taufkirchen, Germany); anti-cytochrome c (7H8.2C12) (BD PharMingen, Heidelberg, Germany); anti-prohibitin (II-14-10) (NeoMarkers, Fremont, CA, USA). Blots were then probed with species-specific horseradish peroxidase-labeled antibodies (Dianova, Hamburg, Germany) and detected with enhanced chemoluminescence (Amersham Biosiences, Freiburg, Germany).

For total protein, SDS-PAGE analysis staining with Coomassie Brilliant Blue (Sigma) was performed after gel electrophoresis.

In vitro phagocytosis of CFSE-labeled $\mathrm{ABs}$ by monocyte-derived phagocytes. After staining with CFSE $(3 \mu \mathrm{M})$ for $10 \mathrm{~min}$ at $20^{\circ} \mathrm{C}$, cells were induced to undergo apoptosis. After $16 \mathrm{~h}$ of incubation, $\mathrm{ABs}$ were isolated from cellular supernatants. Monocytes were isolated from peripheral blood by magneticactivated cell sorting (CD14-positive selection, Miltenyi, Bergisch Gladbach, Germany). These cells were cultured for $24 \mathrm{~h}$ in the presence of GM-CSF (100 $\mathrm{U} / \mathrm{ml}$ ) to emerge monocyte-derived phagocytes. CFSE-labeled ABs were coincubated with monocyte-derived phagocytes (cultured for $24 \mathrm{~h}$ ). Gating on phagocytes, CFSE-fluorescence was analyzed by flow cytometry and increasing fluorescence intensity was detected, if CFSE-labeled ABs had been engulfed.

Microscopy and confocal microscopy. Light microscopy was performed on an Olympus IX70 Inverted Microscope analyzing cells that were transferred into glass-bottom chamber slides using a $\times 100$ oil-immersion lens. For confocal microscopy, cells were harvested and transferred into glass-bottom chamber slides for analysis on a Perkin-Elmer Ultra-View spinning disc confocal on Nikon TE2000-E inverted microscope by using a $\times 100$ oil-immersion lens (excitation at 488,568 , $647 \mathrm{~nm}$, detection at $650 \mathrm{~nm}$, shown red and $488 \mathrm{~nm}$, shown green). For the analysis of phagocytosis, CFSE-stained ABs were co-incubated with monocytederived phagocytes (cultured for $24 \mathrm{~h}$ ), which were stained by a PE-labeled antiCD14 antibody (BD Biosciences, CA, USA).

Transmission electron microscopy. Isolated $A B s$ were fixed by $2 \%$ glutardialdehyde in PBS over night and embedded in small blocks of $1 \%$ agar. ABs were then post-fixed in $1 \%$ osmium tetroxide for $1 \mathrm{~h}$, dehydrated in a graded series of ethanols and embedded in epoxy resin (Epon 812). Semithin and ultrathin sections were cut on an Ultracut E microtome (Leica, Nussloch, Germany). Semithin sections $(1 \mu \mathrm{m})$, stained with toluidine-blue were used for light microscopy. Ultrathin sections $(60-80 \mathrm{~nm})$, stained with uranyl acetate and lead citrate, were observed in a Philips EM 301 (Philips Electronics, Eindhoven, The Netherlands).

Acknowledgements. Confocal microscopy was performed at the Nikon Imaging Center at the University of Heidelberg. We acknowledge Dr. U Engel and Dr. C Ackermann for excellent technical support. Transmission electron microscopy was performed at the Institute of Anatomy and Cell Biology, University of Heidelberg, Germany. We acknowledge Professor Dr. W Kriz, Dr. M Islinger, $\mathrm{H}$ Hosser and B Hähnel for their kind support. HM Lorenz and M Schiller were supported by a research grant from the German Research Foundation (project Lo437/5-3).

1. Gaipl US, Kuhn A, Sheriff A, Munoz LE, Franz S, Voll RE et al. Clearance of apoptotic cells in human SLE. Curr Dir Autoimmun 2006; 9: 173-187.

2. Liu G, Wu C, Wu Y, Zhao Y. Phagocytosis of apoptotic cells and immune regulation. Scand $\mathrm{J}$ Immunol 2006; 64: 1-9.

3. Lorenz HM, Grunke M, Hieronymus T, Winkler S, Blank N, Rascu A et al. Hyporesponsiveness to gammac-chain cytokines in activated lymphocytes from patients with systemic lupus erythematosus leads to accelerated apoptosis. Eur J Immunol 2002 32: $1253-1263$

4. White S, Rosen A. Apoptosis in systemic lupus erythematosus. Curr Opin Rheumatol 2003; 15: $557-562$.

5. Lorenz HM, Grunke M, Hieronymus T, Herrmann M, Kunnel A, Manger B et al. In vitro apoptosis and expression of apoptosis-related molecules in lymphocytes from patients with systemic lupus erythematosus and other autoimmune diseases. Arthritis Rheum 1997; 40: 306-317.

6. Perniok A, Wedekind F, Herrmann M, Specker C, Schneider M. High levels of circulating early apoptic peripheral blood mononuclear cells in systemic lupus erythematosus. Lupus 1998; 7: 113-118.

7. Licht R, Dieker JW, Jacobs CW, Tax WJ, Berden JH. Decreased phagocytosis of apoptotic cells in diseased SLE mice. J Autoimmun 2004; 22: 139-145.

8. Herrmann M, Voll RE, Zoller OM, Hagenhofer M, Ponner BB, Kalden JR. Impaired phagocytosis of apoptotic cell material by monocyte-derived macrophages from patients with systemic lupus erythematosus. Arthritis Rheum 1998; 41: 1241-1250.

9. Gabler C, Blank N, Hieronymus T, Schiller M, Berden JH, Kalden JR et al. Extranuclear detection of histones and nucleosomes in activated human lymphoblasts as an early event in apoptosis. Ann Rheum Dis 2004; 63: 1135-1144.

10. Radic M, Marion T, Monestier M. Nucleosomes are exposed at the cell surface in apoptosis. J Immunol 2004; 172: 6692-6700.

11. Frisoni L, McPhie L, Colonna L, Sriram U, Monestier M, Gallucci S et al. Nuclear autoantigen translocation and autoantibody opsonization lead to increased dendritic cell phagocytosis and presentation of nuclear antigens: a novel pathogenic pathway for autoimmunity? J Immunol 2005; 175: 2692-2701. 
12. Distler JH, Pisetsky DS, Huber LC, Kalden JR, Gay S, Distler O. Microparticles as regulators of inflammation: novel players of cellular crosstalk in the rheumatic diseases. Arthritis Rheum 2005; 52: 3337-3348.

13. Hugel B, Martinez MC, Kunzelmann C, Freyssinet JM. Membrane microparticles: two sides of the coin. Physiology (Bethesda) 2005; 20: 22-27.

14. Pilzer D, Gasser O, Moskovich O, Schifferli JA, Fishelson Z. Emission of membrane vesicles: roles in complement resistance, immunity and cancer. Springer Semin Immunopathol 2005; 27: 375-387.

15. Thery C, Duban L, Segura E, Veron P, Lantz O, Amigorena S. Indirect activation of naive CD4+ T cells by dendritic cell-derived exosomes. Nat Immunol 2002; 3: $1156-$ 1162.

16. Segura E, Guerin C, Hogg N, Amigorena S, Thery C. CD8+ dendritic cells use LFA-1 to capture MHC-peptide complexes from exosomes in vivo. J Immunol 2007; 179 1489-1496.

17. Cocca BA, Cline AM, Radic MZ. Blebs and apoptotic bodies are B cell autoantigens. $J$ Immunol 2002; 169: 159-166.

18. Sebbagh M, Hamelin J, Bertoglio J, Solary E, Breard J. Direct cleavage of ROCK II by granzyme $B$ induces target cell membrane blebbing in a caspase-independent manner. J Exp Med 2005; 201: 465-471.

19. Orlando KA, Stone NL, Pittman RN. Rho kinase regulates fragmentation and phagocytosis of apoptotic cells. Exp Cell Res 2006; 312: 5-15.

20. Lane JD, Allan VJ, Woodman PG. Active relocation of chromatin and endoplasmic reticulum into blebs in late apoptotic cells. J Cell Sci 2005; 118: 4059-4071.

21. Cline AM, Radic MZ. Apoptosis, subcellular particles, and autoimmunity. Clin Immuno 2004; 112: 175-182.

22. Fadeel B. Plasma membrane alterations during apoptosis: role in corpse clearance Antioxid Redox Signal 2004; 6: 269-275.

23. Geske FJ, Monks J, Lehman L, Fadok VA. The role of the macrophage in apoptosis: hunter, gatherer, and regulator. Int J Hematol 2002; 76: 16-26.

24. Voll RE, Herrmann M, Roth EA, Stach C, Kalden JR, Girkontaite I. Immunosuppressive effects of apoptotic cells. Nature 1997; 390: 350-351.

25. Gabler C, Kalden JR, Lorenz HM. The putative role of apoptosis-modified histones for the induction of autoimmunity in Systemic Lupus Erythematosus. Biochem Pharmacol 2003; 66: 1441-1446.
26. Cheung WL, Ajiro K, Samejima K, Kloc M, Cheung P, Mizzen CA et al. Apoptotic phosphorylation of histone $\mathrm{H} 2 \mathrm{~B}$ is mediated by mammalian sterile twenty kinase. Cell 2003; 113: $507-517$

27. Kroemer G, Reed JC. Mitochondrial control of cell death. Nat Med 2000; 6: 513-519.

28. Marchetti P, Hirsch T, Zamzami N, Castedo M, Decaudin D, Susin SA et al. Mitochondrial permeability transition triggers lymphocyte apoptosis. J Immunol 1996; 157: 4830-4836.

29. Martin S, Tesse A, Hugel B, Martinez MC, Morel O, Freyssinet JM et al. Shed membrane particles from $T$ lymphocytes impair endothelial function and regulate endothelial protein expression. Circulation 2004; 109: 1653-1659.

30. Qian Y, Wang H, Clarke SH. Impaired clearance of apoptotic cells induces the activation of autoreactive anti-Sm marginal zone and B-1 B cells. J Immunol 2004; 172: 625-635

31. Radic MZ, Shah K, Zhang W, Lu Q, Lemke G, Hilliard GM. Heterogeneous nuclear ribonucleoprotein P2 is an autoantibody target in mice deficient for Mer, Axl, and Tyro3 receptor tyrosine kinases. J Immunol 2006; 176: 68-74.

32. Heyder P, Bekeredjian-Ding I, Parcina M, Blank N, Ho AD, Herrmann M et al. Purified apoptotic bodies stimulate plasmacytoid dendritic cells to produce IFN-alpha. Autoimmunity 2007; 40: 331-332

33. Lorenz HM, Hieronymus T, Grunke M, Manger B, Kalden JR. Differential role for IL-2 and IL-15 in the inhibition of apoptosis in short-term activated human lymphocytes. Scand J Immunol 1997; 45: 660-669.

34. Hieronymus T, Blank N, Gruenke M, Winkler S, Haas JP, Kalden JR et al. CD 95-independent mechanisms of IL-2 deprivation-induced apoptosis in activated human lymphocytes. Cell Death Differ 2000; 7: 538-547.

35. Thery C, Boussac M, Veron P, Ricciardi-Castagnoli P, Raposo G, Garin J et al. Proteomic analysis of dendritic cell-derived exosomes: a secreted subcellular compartment distinct from apoptotic vesicles. J Immunol 2001; 166: 7309-7318.

36. Riemekasten G, Hahn BH. Key autoantigens in SLE. Rheumatology (Oxford) 2005; 44: 975-982.

37. Nicoletti I, Migliorati G, Pagliacci MC, Grignani F, Riccardi C. A rapid and simple method for measuring thymocyte apoptosis by propidium iodide staining and flow cytometry. $\mathrm{J}$ Immunol Methods 1991; 139: 271-279.

38. Koopman G, Reutelingsperger CP, Kuijten GA, Keehnen RM, Pals ST, van Oers MH. Annexin $\mathrm{V}$ for flow cytometric detection of phosphatidylserine expression on $\mathrm{B}$ cells undergoing apoptosis. Blood 1994; 84: 1415-1420. 